\title{
Florivory as an Opportunity Benefit of Aposematism
}

\author{
Andrew D. Higginson, ${ }^{1, *}$ Michael P. Speed, ${ }^{2}$ and Graeme D. Ruxton ${ }^{3}$
}

1. School of Biological Sciences, Life Sciences Building, University of Bristol, 24 Tyndall Avenue, Bristol BS8 1TQ, United Kingdom; 2. Department of Evolution, Ecology and Behavior, Institute of Integrative Biology, Faculty of Health and Life Sciences, Biosciences Building, University of Liverpool, Crown Street, Liverpool L69 7ZB, United Kingdom; 3. School of Biology, University of St. Andrews, St Andrews KY16 9TH, United Kingdom

Submitted November 21, 2014; Accepted June 11, 2015; Electronically published October 1, 2015

Online enhancements: appendix, zip file.

\begin{abstract}
Inconspicuous prey pay a cost of reduced feeding opportunities. Flowers are highly nutritious but are positioned where prey would be apparent to predators and often contain toxins to reduce consumption. However, many herbivores are specialized to subvert these defenses by retaining toxins for their own use. Here, we present a model of the growth and life history of a small herbivore that can feed on leaves or flowers during its development and can change its primary defense against visual predators between crypsis and warning coloration. When herbivores can retain plant toxins, their fitness is greatly increased when they are aposematic and can consume flowers. Thus, toxin sequestration leading to aposematism may enable a significant opportunity benefit for florivory. Florivory by cryptic herbivores is predicted when toxins are very potent but are at high concentration only in flowers and not in leaves. Herbivores should usually switch to eating flowers only when large and in most conditions should switch simultaneously from crypsis to aposematism. Our results suggest that florivory should be widespread in later instars of small aposematic herbivores and should be associated with ontogenic color change. Florivory is likely to play an underappreciated role in herbivorous insect life histories and host plant reproductive success.
\end{abstract}

Keywords: flower-eating, folivory, camouflage, crypsis, sequestration, toxins.

\section{Introduction}

Many animals avoid attacks by predators by adopting appearances that make them difficult to detect, such as crypsis (Cott 1940) and masquerade (Skelhorn et al. 2010), but these defenses are fundamentally imperfect. Crypsis is particularly adversely affected when an animal is moving (Ioannou and Krause 2009; Hall et al. 2013) or when it alters the local environment, as small herbivores do when consuming leaves (Heinrich and Collins 1983; Mäntylä et al.

\footnotetext{
* Corresponding author. Present address: Centre for Research in Animal Behaviour, College of Life and Environmental Sciences, University of Exeter, Exeter EX4 4QG, United Kingdom; e-mail: adhigginson@gmail.com.

Am. Nat. 2015. Vol. 186, pp. 728-741. (C) 2015 by The University of Chicago. 0003-0147/2015/18606-55906\$15.00. All rights reserved. DOI: $10.1086 / 683463$
}

2008). Crypsis is further impaired if there is bright sunlight and clear lines of sight for passing visual predators, conditions that occur on the periphery of plants (Stamp and Bowers 1988, 1991). This is unfortunate for herbivores because flowers - which plants typically produce in exposed positions - have low physical defenses at anthesis, are often highly nutritious, and so offer potential for rapid herbivore growth (Damman 1987; Stamp and Bowers 1991; van Dam et al. 1996; Held and Potter 2004). Even where herbivores' colors match flowers', they are likely to be more conspicuous than herbivores feeding on interior leaves. Individual plants are therefore a heterogeneous environment with respect to both mortality risk and energy gain, and so herbivores must decide in which microhabitat to forage (Heinrich 1979; Damman 1987; Stamp and Bowers 1988; Reader and Hochuli 2003; Takeuchi et al. 2009) to optimize the trade-off between growth and mortality (Werner and Gilliam 1984; Higginson et al. 2012).

This trade-off may be altered if herbivores subvert plant defenses by retaining plant toxins from their food for defense (Opitz and Müller 2009) and advertise this defense with signals that deter predators (Poulton 1890; Mappes et al. 2005). Toxin-sequestering herbivores sometimes choose plants that contain toxins even when other plants would support faster growth (Singer et al. 2004a, 2004b). The visibility-associated costs of florivory for cryptic prey do not apply to aposematic prey, because predators do not attack them. Therefore, aposematic herbivores can feed freely in clear sight of their predators, such as on flowers. Foraging theory is often concerned with opportunity costs associated with different options (Winterhalder 1983; Nonacs 2001; Eccard and Liesenjohann 2014). Florivory may provide an opportunity benefit of aposematism (Stevens and Ruxton 2013) through enhanced resource collection, which is underappreciated in the understanding of the prevalence and evolution of aposematism (Speed et al. 2010).

Aposematism is often not tenable for small animals because their warning signal is weak and individually they 
expose predators to small, ineffective toxin doses (Forsman and Merilaita 1999; Mänd et al. 2007; Remmel and Tammaru 2011). If the animal is cryptic when small, thenfrom the arguments above - it should feed on interior leaves during initial instars (Smallegange et al. 2007; Agerbirk et al. 2010; Bandeili and Müller 2010). Thus, larval florivores should be later instars, should be chemically defended, and should advertise those defenses. Florivory may therefore be associated with ontogenic color change (OCC), commonly seen in prey animals (Grant 2007; Wilson et al. 2007). On the other hand, accumulating defenses (Bowers 1992, 1993) and eating subprime food in plant interiors (Damman 1987; Stamp and Bowers 1991) will slow herbivore growth and delay maturity. Therefore, a herbivore may benefit from being cryptic against flowers and ingesting their resources quickly, growing quickly and minimizing the duration of the exposure to predators (Benrey and Denno 1997; Williams 1999). Which is the best strategy depends on how the vulnerability of the prey animal varies over its growth period (Benrey and Denno 1997).

Unfortunately, the ecology and behavior of florivores are understudied in most habitats (McCall and Irwin 2006). An exception is Diniz and Morais's (2002) data on the Brazilian cerrado, showing that flowers support the larvae of more lepidopteran species than do leaves, but such florivores are generally less polyphagous than folivores, suggesting specialization to particular hosts (Morais et al. 2009 ). In this environment, larvae of $17 \%$ of 83 lepidopteran species on 36 plants species fed on both leaves and flowers but were specialists on particular host plants and so could switch between leaves and flowers during development. The role of florivory in the evolution of prey defenses has not been considered, despite implications for the evolution of plant defenses. Given the diversity of life-history strategies available to a herbivore, a qualitative model is essential to compare strategies across a range of ecological situations.

Previously, we showed how OCC can arise if size affects detectability and the effectiveness of warning signals (Higginson and Ruxton 2010), and we investigated how accumulating toxin content should influence microhabitat choice (Higginson et al. 2012). However, how these two influences should combine to determine a combined strategy of microhabitat choice and color change has not been elucidated. This makes it difficult to assess how herbivore defenses and plant-herbivore interactions will be influenced by the potential for florivory presented by most host plants. Flowers are available for only a fraction of the vegetative period of most plants and so will not always be available to herbivores, but there may be strong selective advantages to consuming them when available. In order to assess the greatest potential advantage of florivory, we assume in our model that flowers are always available for the growth period of the herbivore. We use dynamic programming of insect life history to find the fitness advantages of a combination of florivory and aposematism relative to only crypsis and/or folivory. We characterize a herbivore, in line with Smallegange et al. (2007), not as sequestering toxins but rather as storing them for responsive defenses (Higginson and Ruxton 2009), such as the regurgitation exhibited by many lepidopteran larvae (Grant 2006). For example, Pieris brassicae stores highly concentrated glucosinolate hydrolysis products in the foregut (Smallegange et al. 2007) that are costly to use (Higginson et al. 2011; Daly et al. 2012). The relative nutrition and toxicity of flowers, compared to those of leaves, varies greatly (McCall and Irwin 2006), and so we explored the impact of this variability for the evolution of florivory.

We find that toxin potency, leaf toxicity, and warningsignal effectiveness all affect whether florivory should evolve. We show that florivory could be a strong opportunity benefit to an aposematic lifestyle in a broad range of circumstances, which helps explain the ubiquity of aposematism among small herbivores and suggests that florivory should be common when it is possible. We make clear predictions for the conditions under which florivory will be observed among cryptic herbivores. Florivory has an increasingly well-recognized influence on ecosystem dynamics through effects on plant reproductive success (McCall and Irwin 2006), and our model suggests that florivory might also play an important role in herbivore-plant defense coevolution.

\section{The Model}

We model an environment comprised of two habitats, leaves (L) and flowers (F), that differ in their energy concentration ( $r$, the mass per unit mass of tissue), toxin concentration ( $g$, the mass of toxin per unit mass of tissue), and apparency to predators $d$ (see table 1 for definitions of all parameters and variables). We set energy and toxin concentrations in flowers to unity $\left(r_{\mathrm{F}}=g_{\mathrm{F}}=1\right)$, so that energy concentration in leaves is a proportion $r_{\mathrm{L}}$ of that in flowers and, similarly, toxin concentration in leaves is a proportion $g_{\mathrm{L}}$ of that in flowers. Given data presented in McCall and Fordyce (2010), we assume that leaves are lower in both toxins and energy than flowers $\left(0 \leq r_{\mathrm{L}} \leq 1\right.$, $0 \leq g_{\mathrm{L}} \leq 1$, and $g_{\mathrm{L}}=r_{\mathrm{L}}=0.7$ in the baseline parameterization). A small herbivore must use gathered energy and toxins to grow, defend itself, and mature at the size and time under its control so as to maximize its fitness, here defined as the product of the probability of survival to maturation and the reproductive value at maturation. That is, the herbivore trades off mortality during growth against the benefits of large size after maturation (Higginson and Ruxton 2010). 
Table 1: Parameters and variables used in the model and their default values

\begin{tabular}{|c|c|c|}
\hline Parameter & Symbol & Value \\
\hline \multicolumn{3}{|l|}{ State variables and parameters: } \\
\hline Body mass & $x$ & $\ldots$ \\
\hline Toxin load & $y$ & $\cdots$ \\
\hline Coloration strategy & $z$ & $\ldots$ \\
\hline Feeding location & $u$ & $\ldots$ \\
\hline Maximum body mass & $X_{\max }$ & 1,000 \\
\hline Maximum toxin load & $Y_{\max }$ & 50 \\
\hline Midpoint of size-fecundity relation & $c$ & 500 \\
\hline Slope of size on fecundity relation & $b$ & .02 \\
\hline \multicolumn{3}{|l|}{ Food consumption parameters: } \\
\hline Energy concentration of leaves & $r_{\mathrm{L}}$ & .7 \\
\hline Energy concentration of flowers & $r_{\mathrm{F}}$ & 1.0 \\
\hline Toxin concentration of leaves & $g_{\mathrm{L}}$ & .7 \\
\hline Toxin concentration of flowers & $g_{\mathrm{F}}$ & 1.0 \\
\hline Scalar of body mass on food consumption & $\sigma$ & $2 / 3$ \\
\hline Scalar of food consumption & $\gamma$ & .05 \\
\hline Potency of toxin & $\theta$ & .05 \\
\hline \multicolumn{3}{|l|}{ Predation parameters: } \\
\hline Relative detectability when cryptic on leaves & $d_{\mathrm{C}, \mathrm{L}}$ & .025 \\
\hline Relative detectability when cryptic on flowers & $d_{\mathrm{C}, \mathrm{F}}$ & .1 \\
\hline Relative detectability when aposematic on leaves & $d_{\mathrm{A}, \mathrm{L}}$ & .8 \\
\hline Relative detectability when aposematic on flowers & $d_{\mathrm{A}, \mathrm{F}}$ & 1.0 \\
\hline Predator density & $\mu$ & .01 \\
\hline Relative predation risk when switching & $\mu_{\mathrm{S}}$ & 10 \\
\hline Warning-signal effectiveness & $\psi$ & .25 \\
\hline Toxin benefit ratio & $\rho$ & 1.0 \\
\hline Aversion to aposematism combination & $\lambda$ & 1.0 \\
\hline
\end{tabular}

The herbivore is characterized by four state variables that may change at each time step $t$ : body mass $x_{t}$, toxin load $y_{t}$, coloration strategy $z_{t}$, and location within the host plant $u_{t}$. The herbivore may be currently feeding on either leaves $\left(u_{t}=\mathrm{L}\right)$ or flowers $\left(u_{t}=\mathrm{F}\right)$ and may be either conspicuous $\left(z_{t}=\mathrm{A}\right)$ or cryptic against its current background (leaves or flowers; $z_{t}=\mathrm{C}$ ). At each time step, the herbivore makes two decisions: an action $\alpha$ and a toxin-retention decision $\delta$. Decision $\alpha$ takes one of five possibilities: (1) mature (e.g., pupate), that is, cease feeding and receive the fitness payoff $W(x)$ (see eq. [6] below); (2) change locations ( $u$ changes); (3) change coloration ( $z$ changes); (4) change both location and coloration; and (5) feed. When changing location and/or coloration, the herbivore cannot feed but is still at risk of predation, which is increased by a factor of $\mu_{\mathrm{s}}$. Thus, (modest) costs accrue from changing appearance or location. Note that we assume that if the herbivore switches location but remains cryptic, it changes color simultaneously to be cryptic on the new background.

When feeding, a herbivore ingests a mass of plant tissue per time step that scales with body mass. Specifically, the herbivore ingests a mean amount of tissue $\gamma x^{\sigma}$, where $\gamma$ and $\sigma$ are dimensionless quantities that control the rela- tion of body mass to food consumption (we assume some variation in food intake to avoid grid effects in computations; Houston and McNamara 1999). We assume $\sigma<1$ so that growth is decelerating, as is characteristic of insect herbivores (Tammaru and Esperk 2007). Since our focus is on the role of toxins, we use "energy" as a proxy for all nutrients used in growth and metabolism (i.e., "energy" has mass). When the herbivore feeds, it ingests toxins that impose an immediate energetic cost (e.g., gut processing; Bowers 1992) of $g_{\mathrm{L}} \theta$ per unit of ingested leaf tissue or $g_{\mathrm{F}} \theta$ per unit of ingested floral tissue, where $\theta$ is a (dimensionless) toxin potency. We assume that a herbivore cannot synthesize toxins. Instead, decision $\delta$ controls the proportion of the toxin load that the herbivore retains in its body (being neither excreted nor destroyed). Maintaining toxins as defenses imposes an energy cost (e.g., preventing autotoxicity; Bowers 1992). Thus, the herbivore pays an unavoidable cost of eating toxins but pays further costs only if toxins are not immediately discarded. Note that we implicitly assume a conversion between the energetic cost of toxins and their mass, such that toxin load makes up a negligible proportion of total body mass. We assume negligible energetic costs of foraging and growth, which is reasonable 
for ectothermic larvae (Reynolds et al. 1985; Tammaru and Esperk 2007). Thus, the net gain in energy is converted directly into body mass. Given the above assumptions, if the herbivore feeds, then its mass $x$ at the next time step is

$$
x_{t+1}=x_{t}+\gamma x_{t}^{\sigma}\left(r_{i}-g_{i} \theta\right)-\theta y_{t}
$$

where $i \in\{\mathrm{L}, \mathrm{F}\}$. That is, body mass at the next time step equals body mass at the previous time step plus the net energy gain after toxin consumption and maintenance costs are met. We assume that during each time step eating occurs before any predator attacks, so the toxicity of the herbivore, $y$, before any attack is

$$
y_{t+1}=\delta\left(y_{t}+\gamma x_{t}^{\sigma} g_{i}\right),
$$

where $i \in\{\mathrm{L}, \mathrm{F}\}$. That is, toxin load is increased by the amount of food eaten multiplied by the toxin concentration in the food, and then some proportion of the total is retained. If the herbivore does not feed (i.e., $\alpha \neq 5$ ), then $\gamma=0$ in equations (1) and (2). From computational necessity, we assume that $x$ and $y$ are limited to not exceed $X_{\max }$ and $Y_{\max }$, respectively, but preliminary evaluations of the strategy enabled us to ensure that these values were sufficiently large that they do not affect the optimal strategy.

The herbivore is at risk from predators for all activities unless it matures (i.e., for $\alpha \neq 1$ ). The probability of being detected per time step $D$ depends on size $x$, predator density $\mu$, coloration strategy $z$, and location $u$. We assume that the probability of detection increases with body mass proportionally to surface area (as in Berger et al. 2006); thus,

$$
D=d_{j, i} x_{t}^{2 / 3} \mu,
$$

where $i \in\{\mathrm{L}, \mathrm{F}\}$ and $j \in\{\mathrm{C}, \mathrm{A}\}$. Here, $\mu$ is the probability of detection per unit area and $d_{j, i}$ reduces the probability proportionally, depending on location and appearance. We assume that cryptic prey are less detectable by predators than conspicuous prey on both leaves and flowers $\left(d_{\mathrm{C}, i}<d_{\mathrm{A}, i}\right)$ but that predators detect prey more frequently when they are on flowers $\left(d_{j, \mathrm{~L}}<d_{j, \mathrm{~F}}\right)$. We make the latter assumption because flowers are on the periphery of plants and so predators will have clear lines of sight. An aposematic signal could be less conspicuous on flowers $\left(d_{\mathrm{A}, \mathrm{F}}<d_{\mathrm{A}, \mathrm{L}}\right)$ because of partial background matching (e.g., yellow-black caterpillars on yellow flowers), but this would clearly increase the benefit for only warningly colored florivory, so this assumption makes the model a conservative characterization of the fitness benefits of this strategy.

The probability of a predator attacking $(A)$ a conspicuous prey declines with the body surface area because larger warning signals are more effective, according to

$$
A=\frac{1}{1+x_{t}^{2 / 3} \psi}
$$

where $\psi$ is the effectiveness of warning coloration per unit area. The particular form of $A$ does not affect predictions, provided that $A$ decreases with $x$ at a decelerating rate, as expected from observations on lepidopteran larvae (Mänd et al. 2007). If the prey is cryptic, then $\psi=0$, so the predator always attacks (Mänd et al. 2007).

If a herbivore is attacked, we assume that half of the current toxin load $y_{t+1}$ is lost in defense, on the basis of observations for Pieris brassicae (Higginson et al. 2011; Daly et al. 2012) and our intuition that herbivores conserve some toxin to defend against future attacks. The probability that a predator kills a herbivore declines with the product of the potency of the toxin $\theta$ and the toxin effectiveness ratio $\rho$, and this effect is greater for conspicuous prey by $\lambda(\lambda>0)$, because toxins are more effective when paired with warning coloration (Ruxton et al. 2004). Thus, the probability of mortality if a predator attacks a conspicuous prey is given by

$$
M=e^{-y_{t+1} \theta \rho(1+\lambda)},
$$

where $\rho$ is the effectiveness per $\theta(1+\lambda)$ units of toxin and $\lambda$ is a dimensionless quantity. Thus, we assume that toxins defend against predators with diminishing returns. Again, the choice of the particular form of this equation does not affect the results, provided that the relation decelerates. If a herbivore is undefended $\left(y_{t+1}=0\right)$, it is definitely killed. If the prey is not conspicuous, $\lambda$ takes the value 0 in equation (5). The probability of mortality per time step is the product $D \cdot A \cdot M$.

If a herbivore matures, its reproductive value depends on its size, according to

$$
W(x)=\frac{1}{1+e^{b\left(c-x_{t}\right)}},
$$

where $b$ and $c$ are dimensionless scalars that control how reproductive value increases with body mass. Under the baseline parameter values, reproductive value is negligible for $x<200$ and approaches unity for $x>800$ (based on data in Berger et al. 2006).

Given the above assumptions, we use state-dependent dynamic programming (appendix, available online; McNamara 1990; Houston and McNamara 1999) to find the optimal decisions for integer values of the states that maximize future reproductive value: $\alpha(x, y, z, u)$ and $\delta(x, y, z, u)$. The optimal strategy for the baseline parameter values is presented in the appendix. We then simulate 20 replicates of 1,000 individuals implementing this strategy to calculate the emergent strategy (i.e., retention of toxins and switches between locations and coloration strategy); the mean size, defense level, and age at maturation; the probability of surviving to maturation; and the reproductive value at maturation. The product of the latter two variables is proportional to the selection on the herbivore and is termed "fitness." We com- 
pare the results of the optimal strategy to those for individuals constrained by an inability to feed on flowers $(u \neq 1)$ and/or be warningly colored $(z \neq 1)$ to assess the separate and combined benefits of florivory and aposematism.

\section{Results \\ Details of Herbivore Strategies}

Figure 1 shows the growth and mortality rates for the range of body mass and the baseline parameter values (table 1). Growth increases with body mass $x$ and decreases with the concentration of toxic defenses $y$ and so is always fastest for cryptic (nontoxic) herbivores on flowers and slowest for aposematic (mildly toxic) herbivores on leaves (fig. 1a). Growth is negative when herbivores are very small and aposematic because of the costs of maintaining toxins. Growth increases more rapidly with body mass when the herbivore is eating flowers rather than leaves, and so there is a size above which growth of aposematic herbivores on flowers exceeds that of cryptic herbivores on leaves. Mortality risk increases steeply with body mass for cryptic prey, because crypsis is degraded by large size, whereas for aposematic prey the risk increases only slightly with increasing body mass, because the increased effectiveness of the warning signal compensates for increased apparency (fig. 1b). Therefore, the difference in mortality rate between folivores and florivores is much larger when they are cryptic than when they are aposematic. In contrast, the difference in growth between folivores and florivores is the same whether they are cryptic or aposematic (fig. 1a). Thus, at all sizes, the ratio of growth rate to mortality rate is higher on flowers than on leaves for aposematic herbivores, whereas the opposite holds for cryptic herbivores (fig. 1c). Therefore, herbivores should be either cryptic on leaves or aposematic on flowers for the baseline parameter values (table 1 ). The ratio of growth to mortality risk is constant for cryptic herbivores, because food intake and mortality rate scale similarly with size, whereas the aposematic signal means that mortality rate increases less with size than does growth rate. Hence, the ratio is greatest (indicating the best option; Werner and Gilliam $1984)$ for cryptic herbivores foraging on leaves when small $(x<380)$ and for warningly colored herbivores foraging on flowers when large $(x \geq 380)$.

Maximization of the ratio of growth rate to mortality rate maximizes fitness only if decisions do not affect future states (Houston and McNamara 1999). Since toxins must be gradually accumulated, herbivore strategies may deviate from these predictions. Figure 2 shows the ranges of growth and survivorship trajectories from the dynamic state-dependent model for the baseline parameterization (table 1). Most mortality occurs when herbivores are small
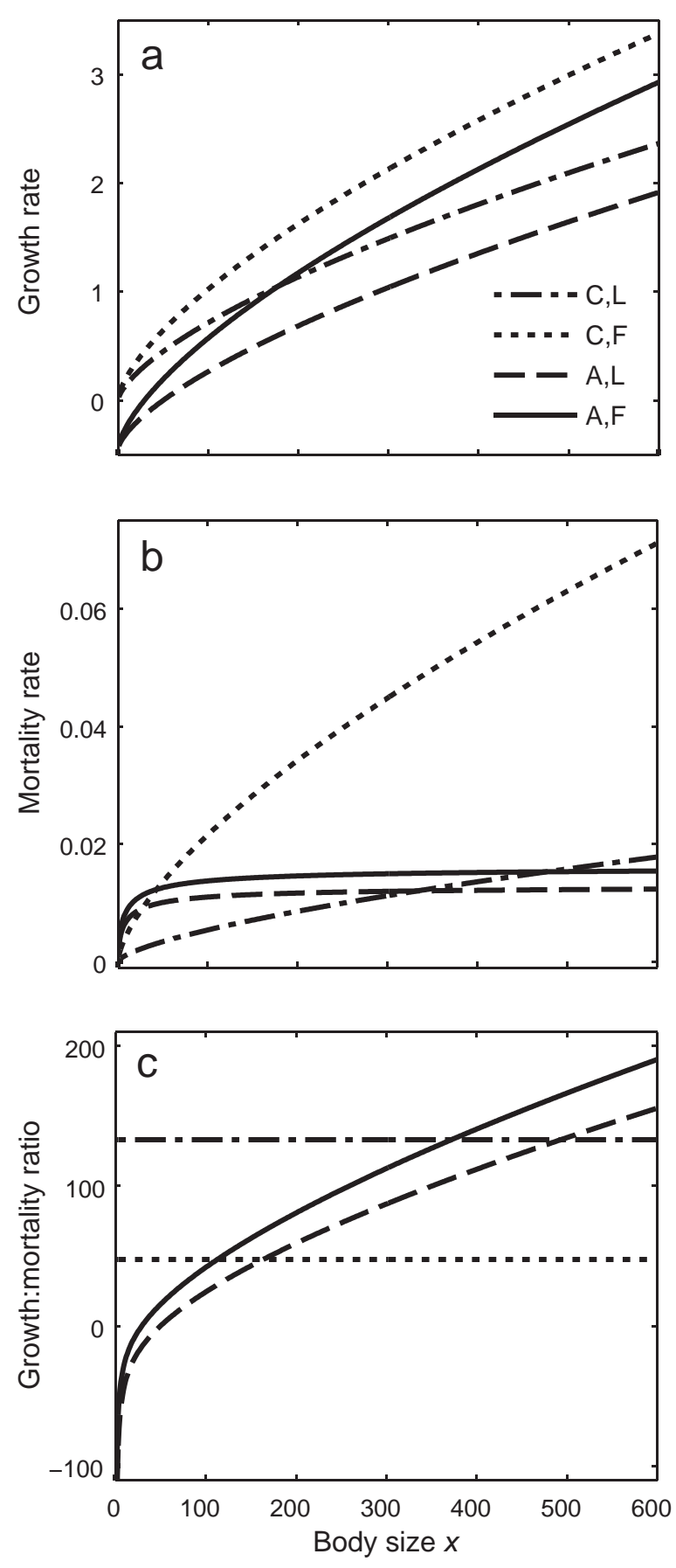

Figure 1: Effects of body mass ( $x$, shown on $X$-axis), location $(u)$, and coloration $(z)$ on growth $(a)$, probability of mortality $(b)$, and their ratio $(c)$. Location and coloration are shown by the lines (dotdashed lines for cryptic folivore: $u=\mathrm{L}, z=\mathrm{C}$; dashed lines for warningly colored folivore: $u=\mathrm{L}, z=\mathrm{A}$; dotted lines for cryptic florivore: $u=\mathrm{F}, z=\mathrm{C}$; solid lines for warningly colored florivore: $u=\mathrm{F}$, $z=\mathrm{A})$. Values shown are for nontoxic $(y=0)$ cryptic prey and toxic $(y=10)$, warningly colored prey. Parameters are as shown in table 1. 


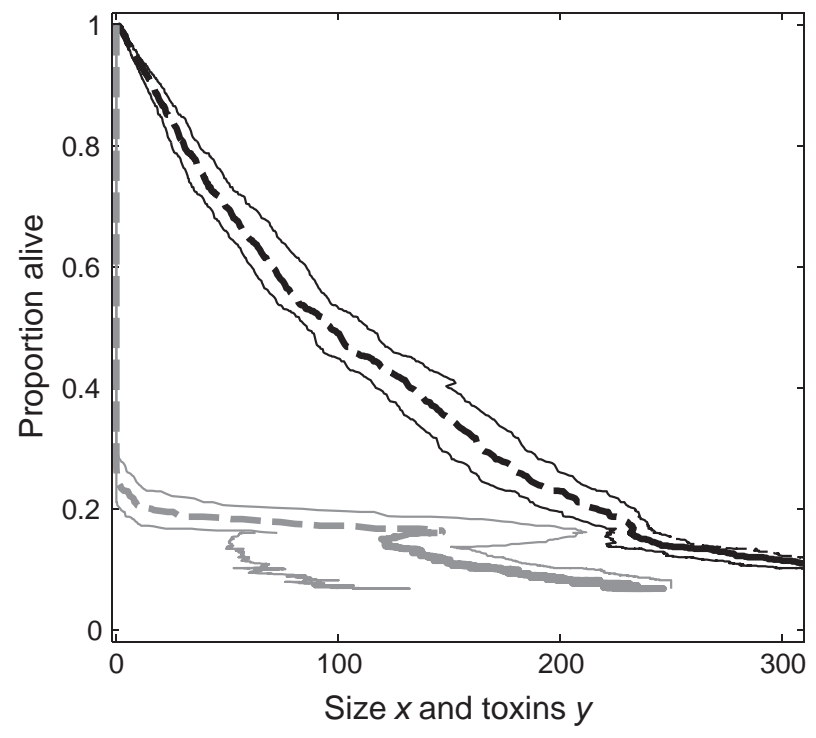

Figure 2: Mean and range of trajectories of body mass ( $x$, black lines) and toxin content ( $y$, gray lines) and the proportion of surviving individuals for the optimal strategy, given the default parameter values. Dashed lines identify when most individuals feed on leaves, and solid lines indicate feeding on flowers. Thin lines indicates maximum and minimum $x$ and $y$ values. All parameters are at the default values shown in table 1 .

and defenseless on leaves (this remains the case even for small values of the parameter that controls the detection risk of cryptic prey on leaves, $d_{\mathrm{C}, \mathrm{L}}$ ). A herbivore should simultaneously switch coloration and switch to florivory at intermediate size $(x \approx 220)$ after it has accumulated some toxins $(y \approx 30)$. This switch should occur at a body mass smaller than expected from figure 1 (i.e., $x=380$ ), because the state-dependent model accounts for the future states, promoting toxin accumulation while the herbivore is still eating leaves to prepare for the higher attack rate on flowers.

\section{Effects of the Differences between Leaves and Flowers}

The relative toxicity of leaves, compared to flowers, varies greatly among defended plants (McCall and Fordyce 2010), so we first explore the impact of the relative toxicity of leaves $\left(g_{L}\right)$ on the optimal and constrained strategies (fig. $3 a, 3 c, 3 e$ ). When florivory and warning coloration are not possible (dot-dashed lines in fig. 3), increased $g_{\mathrm{L}}$ decreases size at maturation (fig. $3 a$ ) and delays maturation (fig. $3 c$ ), because toxin processing retards growth when small (except when $g_{\mathrm{L}}$ is very low). Retention of defenses increases with $g_{\mathrm{L}}$ (not shown), but only slightly, and so does not compensate for the longer exposure to predation; so survival and fitness decline with increasing $g_{\mathrm{L}}$ (fig. $3 e$ ).
When florivory but not warning coloration is possible (dotted lines), switching to flowers is not optimal at any $g_{\mathrm{L}}$. As a result, the age, size, and fitness are the same as for when neither florivory nor warning coloration is possible (fig. $3 a$, $3 c, 3 e)$.

However, if a herbivore can be warningly colored and eat flowers (solid lines), it should switch to this joint state, enabling faster growth when relatively invulnerable and so greatly increasing fitness. Nevertheless, fitness is still maximized if $g_{\mathrm{L}}$ is low, at an intermediate maximum determined by a combination of a negative and a positive effect of increasing $g_{\mathrm{L}}$. The negative effect is that survival in small prey decreases as $g_{\mathrm{L}}$ increases, because growth is slowed when the herbivore is small and toxins are at a high concentration. The positive effect arises from the ability to invest in toxins and be aposematic, so that the herbivore spends more time on flowers, matures sooner, and suffers less mortality risk overall. For warningly colored folivores (dashed lines), fitness also peaks at intermediate leaf toxicity, albeit at a $g_{\mathrm{L}}$ higher than that when florivory is possible. In this case, size at maturity strongly increases with $g_{\mathrm{L}}$ because strong investment in toxicity enables delayed maturation to get the benefit of a large mass at maturation without much increase in mortality.

The relative energy content of leaves, compared to flowers, varies considerably among plants (Held and Potter 2004). If plants conform to optimal-defense theory (McCall and Fordyce 2010), nutrient and toxin concentrations will be positively correlated. Hence, we again vary $g_{\mathrm{L}}$ but also alter the energy concentration of leaves, $r_{\mathrm{L}}$ (fig. $3 b, 3 d, 3 f$ ). When florivory and warning coloration are not possible (dot-dashed lines), the faster growth allowed by elevated $r_{\mathrm{L}}$ increases size at maturation (fig. $3 b$ ) and hastens maturation (fig. $3 d$ ), and so fitness increases despite the increased toxin costs (fig. $3 f$ ). Again, florivory is not optimal when warning coloration is not possible (dotted lines). Warning coloration is highly advantageous at high $r_{\mathrm{L}}$ and $g_{\mathrm{L}}$ (dashed lines), because the best strategy is to invest heavily in defenses, which are more effective when paired with warning coloration. This strategy is optimal even though it incurs a cost of delaying maturation, because mortality rate is greatly reduced and a very large size can be reached. As expected, florivory is most beneficial for intermediate $r_{\mathrm{L}}$ and $g_{\mathrm{L}}$ (fig. $3 f$ ), because age at maturation can decrease as $r_{\mathrm{L}}$ increases when the greater energy content of flowers can be exploited. Thus, florivory and warning coloration should be most common when toxins and/or nutrients are more abundant in flowers than in leaves, but not extremely so.

\section{Effects of Toxin Potency and Concentration}

The proportion of the growth period that herbivores spend on flowers increases with the toxin benefit ratio $(\rho)$ if toxin 

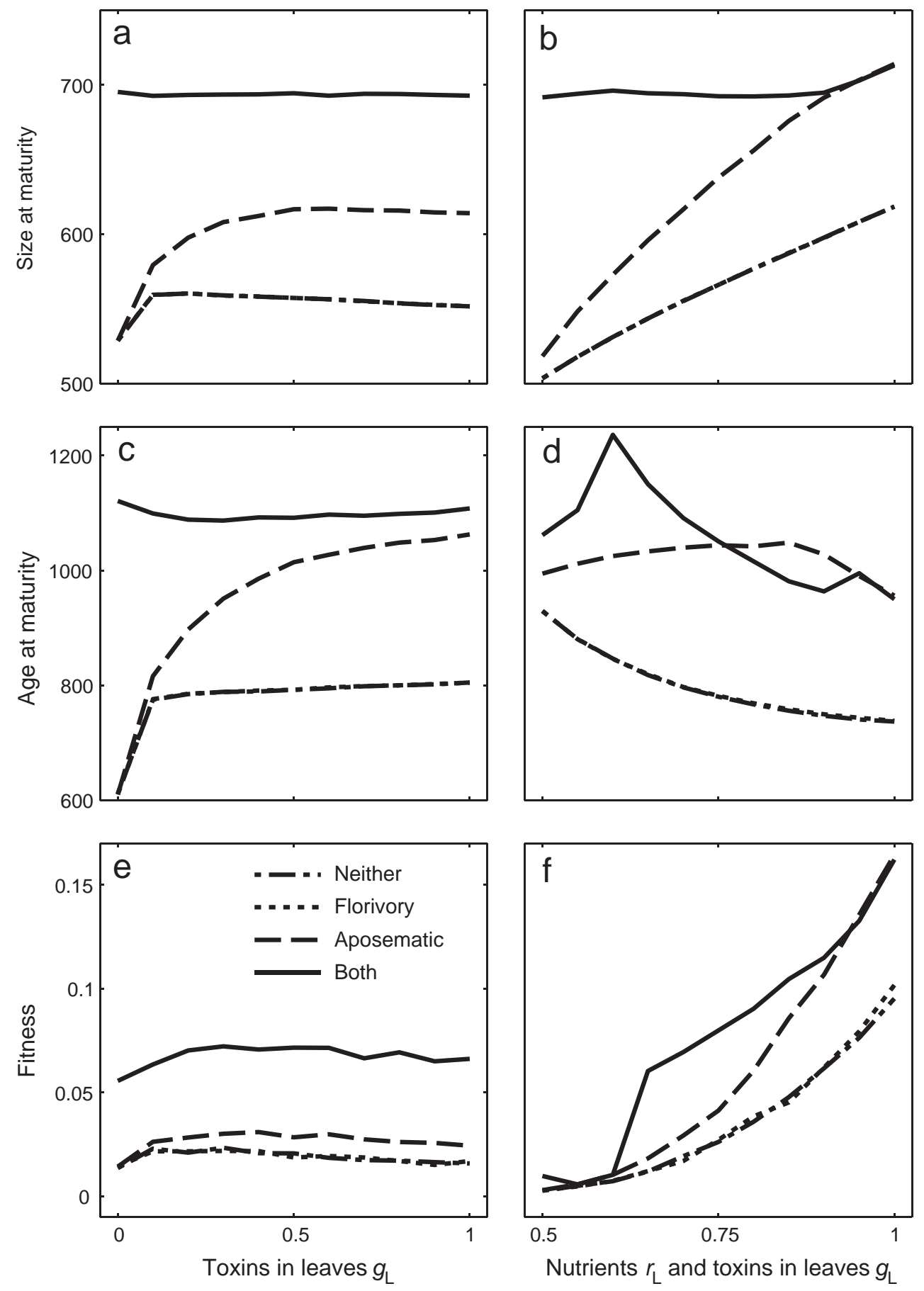

Figure 3: Effects of the toxin content of leaves $g_{\mathrm{L}}(a, c, e)$ and of both the nutrient $\left(r_{\mathrm{L}}\right)$ and toxin $\left(g_{\mathrm{L}}\right)$ contents of leaves $(b, d, f)$ on size at maturity $x^{*}(a, b)$, age at maturity $t^{*}(c, d)$, and fitness (i.e., fecundity $\times$ survivorship; $\left.e, f\right)$ for the optimal strategy under four conditions: full flexibility (i.e., both florivory and aposematism possible; solid lines); the herbivore cannot eat flowers or be warningly colored (dot-dashed lines); the herbivore can eat flowers but cannot be warningly colored (dotted lines); the herbivore can be warningly colored but cannot eat flowers (dashed lines). All other parameters are at the default values shown in table 1. 
potency $\theta$ is low but decreases with $\rho$ if $\theta$ is high (fig. $4 a$ ). The optimal strategy is to switch simultaneously to warning coloration and flower feeding (fig. $4 c$ ) for all these values of $\theta$ and $\rho$. Aposematic florivory is most prevalent when toxins have intermediate potency, because less potent toxins provide poor defense and very potent toxins are too costly to consume. Moving to flowers and becoming warningly colored do not coincide when leaves are almost as nutritious and well defended as flowers, because the proportion of time spent eating flowers becomes low (fig. $4 b$ ), whereas the proportion of time as warningly colored is less sensitive to $r_{\mathrm{L}}$ and $g_{\mathrm{L}}$ (fig. $4 d$ ). Low $r_{\mathrm{L}}$ and $g_{\mathrm{L}}$ promote a period of being warningly colored on leaves, provided that the warning coloration is sufficiently effective. Extensive florivory is expected only when flowers are more nutritious and better defended and predators respond to aposematic signals.

\section{Selective Pressure for Florivory and Aposematism}

We now explore the strength of the benefits of florivory and warning coloration. When florivory and warning coloration are not possible (fig. $5 a, 5 b$ ), fitness is highest for weak toxins (low $\theta$ ), high toxin benefit ratio $\rho$, and effective crypsis (low $d_{\mathrm{C}, \mathrm{L}}$ ) and is obviously unaffected by the effectiveness of crypsis on flowers, $d_{\mathrm{C}, \mathrm{F}}$. Provided that $\theta$ is not very high, the fitness advantage of warning coloration (without florivory) is greatest for a high toxin benefit ratio (high $\rho$; fig. $5 c$ ). Surprisingly, switching to warning
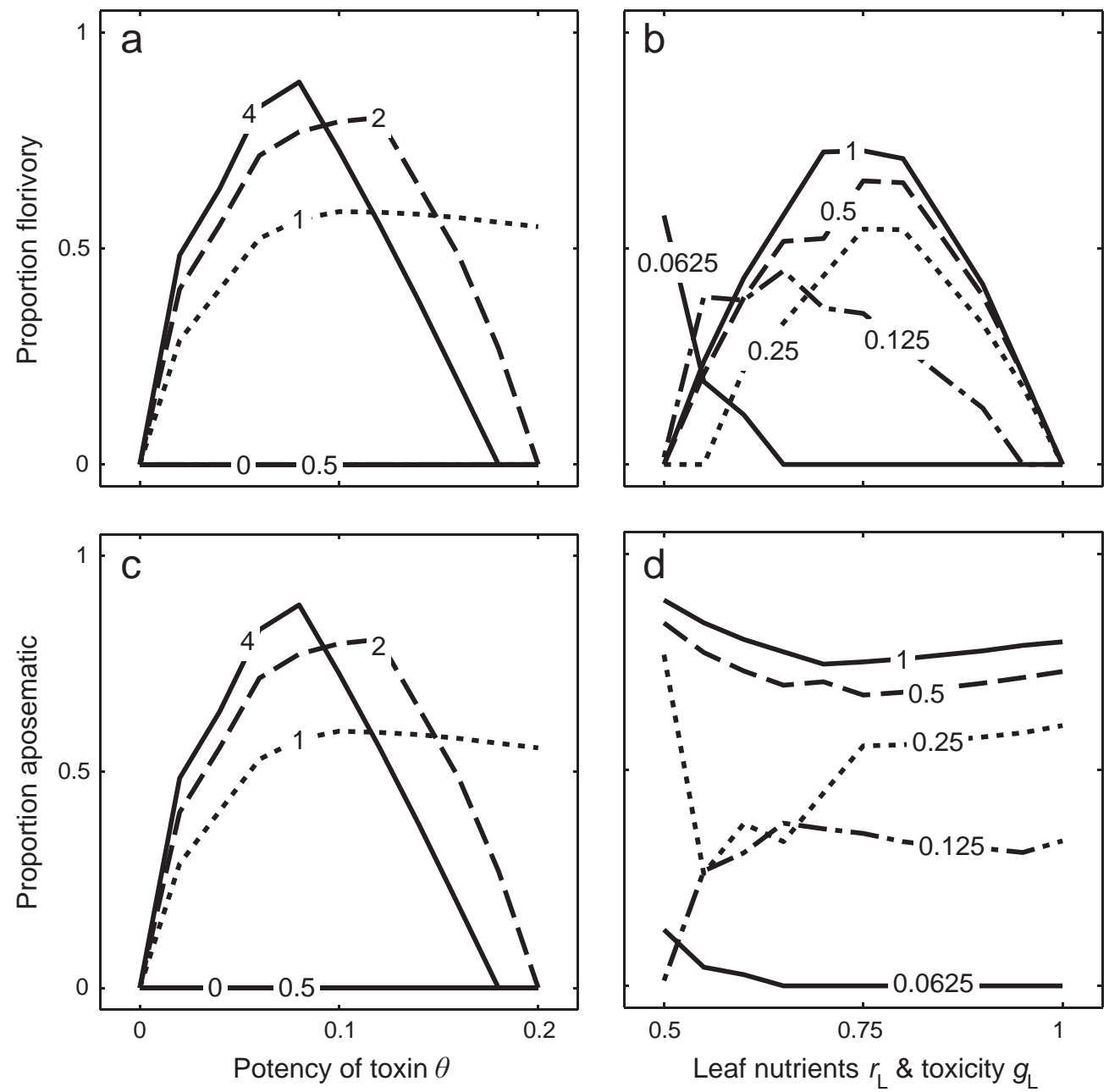

Figure 4: Proportion of the growth period spent as florivorous $(a, b)$ and warningly colored $(c, d)$ for the optimal strategies. We present the effects of potency of the toxin level $\left(\theta, X\right.$-axes) and toxin benefit ratio $(\rho$, lines; $a, c)$, and the nutrient and toxin content of leaves $\left(r_{\mathrm{L}}\right.$ and $g_{\mathrm{L}}$, respectively; $X$-axes) and the signal effectiveness ( $\psi$, lines; $c, d)$. All other parameters are at the default values shown in table 1 . 

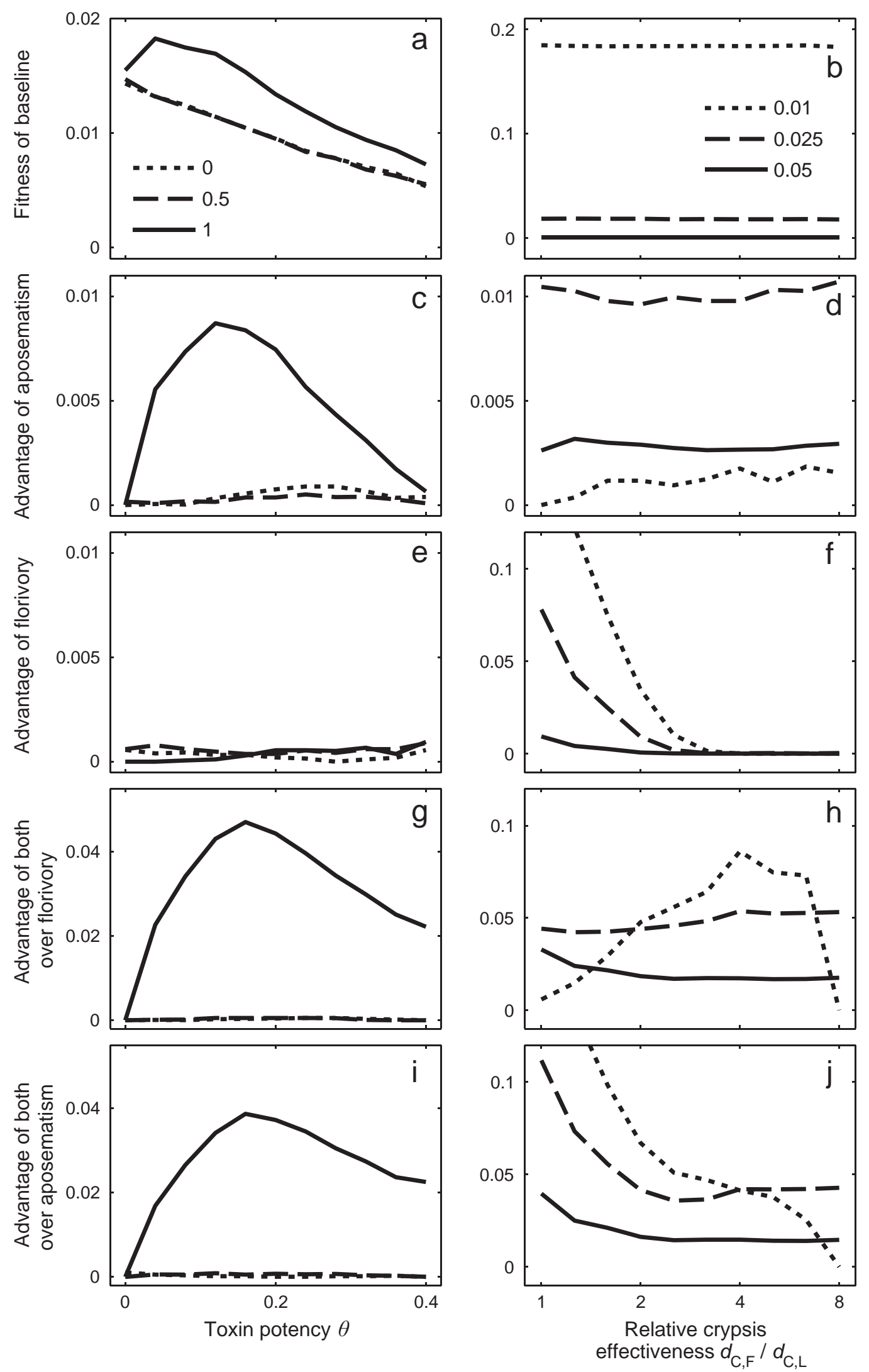

This content downloaded from 138.251.149.242 on February 22, 2017 03:48:55 AM 
coloration is most beneficial (fig. $5 d$ ) when crypsis is mildly effective (intermediate $d_{C, \mathrm{~L}}$ ). This occurs because not suffering attacks when small enables a herbivore to be highly defended when it switches to being warningly colored, but if crypsis on leaves is very effective, then the benefit is smaller because the switch to flowers occurs much later. Again, there is no advantage to florivory without warning coloration unless crypsis on flowers is almost as effective as crypsis on leaves (fig. $5 e, 5 f$ ).

Warning coloration and florivory generally increase fitness synergistically (fig. $5 g, 5 i$ ), provided that the toxin is not very weak (not low $\theta$ ) and the toxin benefit ratio is significant (not low $\rho$ ). This is the case regardless of the detection risk for florivores, provided that $d_{\mathrm{C}, \mathrm{F}} / d_{\mathrm{C}, \mathrm{L}}$ is neither unity nor very high (fig. $5 h$ ). The combination of warning coloration and florivory greatly increases fitness over just aposematism (fig. $5 i, 5 j$ ) under similar conditions, but the advantage declines as the detectability on flowers and the detectability on leaves increase (fig. $5 j$ ). Significantly increasing the aversion of predators to the aposematism combination $(\lambda)$ can increase the survivorship to unrealistically high levels, whereas for lower values, the optimal strategy is to stay on leaves and be cryptic (not shown). Thus, it is critical for our predictions that toxins are more effective for warningly colored prey than for cryptic prey, but otherwise the value of $\lambda$ does not affect our other predictions. For potent but costly toxins and warning colors that are effective against predators, florivory should evolve from aposematic folivory, regardless of the toxicity of leaves (fig. 3). The model predicts the evolution of cryptic florivory only when crypsis on flowers is as effective as crypsis on leaves (fig. $5 f$ ). However, there remains selection to evolve aposematism (fig. $5 h$ ). Even when the best strategy is to eat flowers during the whole period, there is a switch to aposematism and the same investment in toxins as under the strategy at the baseline parameter values.

These predictions about the effects of plant characteristics on the optimal strategy are not qualitatively affected by the particular values of the two parameters describing the size-fecundity relationship ( $c$ and $b$ ) and the parameters controlling food consumption rate ( $\gamma$ and $\sigma$ ). The values of predation risk $\mu$ and the risk of switching $\mu_{\mathrm{S}}$ were chosen on the basis of preliminary model evaluation to ensure low but nonzero survival, as expected for insect (or other small) herbivores.

\section{Discussion}

The results of the model reveal three important points concerning the evolution of aposematism and florivory: (1) if the ability to deploy diet-derived toxins in defense evolves among herbivores, there will be strong selection for warning coloration, (2) aposematic herbivores will be selected to consume flowers whenever they are available, and (3) the conditions for the evolution of florivory among nonaposematic herbivores are restricted. The first point reflects that warning coloration generally is more advantageous than crypsis for large herbivores. Relating to the second point, our model suggests that once toxin use and aposematism evolve, florivory will be highly beneficial. The third point is demonstrated by the restricted parameter space in our model favoring florivory where there is not also selection for warning coloration. More broadly, plant and herbivore defense strategies can influence the extent to which slow growth elevates herbivore mortality (Benrey and Denno 1997) and therefore have long-term positive impacts on plants via reduced herbivore abundance in subsequent generations. Given the range of herbivore strategies, coevolutionary models are needed to explore the consequences of such intergeneration effects for plant-herbivore coevolution. Our work should guide the development of such models.

Although we have described the model in terms of florivory, in many plants young leaves share many of the key features of flowers depicted by our model: a very different color from mature leaves (e.g., delayed greening; Kursar and Coley 1992), reliance on chemical rather than physical defenses (Herms and Mattson 1992), high nutritional value (Herms and Mattson 1992), and positioning on the periphery of plants. Thus, predictions concerning florivory may often be valid for herbivory of new leaves. The great variation observed in the relative defense levels of old leaves, new leaves, and flowers (McCall and Fordyce 2010) provides an opportunity to test the predictions of our model. To simplify the modeling, we consider feeding herbivores to require a single nutrient for their growth. In

Figure 5: Comparisons of the fitness (survival $\times$ reproductive value) under the four flexibility conditions. We present results for combinations of four critical parameters: the potency of the toxin $\theta$ (X-axis) and the toxin benefit ratio $\rho$ (0: dotted lines, 0.5 : dashed lines, or 1: solid lines; $a, c, e, g, i)$; and the ratio of effectiveness of crypsis on flowers to crypsis on leaves (X-axis) and the detection rate of cryptic herbivores on leaves $d_{\mathrm{C}, \mathrm{L}}(0.01$ : dotted lines, 0.025 : dashed lines, or 0.05 : solid lines; $b, d, f, h, j) . a$ and $b$ show the fitness of the cryptic-only, folivory-only strategy. Other panels show comparisons between flexibility conditions as follows: $c, d$, fitness benefit of aposematism compared to the cryptic-only, folivory-only strategy; $e, f$, fitness benefit of florivory compared to the cryptic-only, folivory-only strategy; $g$, $h$, fitness benefit of combined florivory and aposematism compared to the florivory-only strategy; and $i, j$, fitness benefit of combined florivory and aposematism compared to the aposematism-only strategy. Note the variable scales on $Y$-axes. All other parameters are at the default values shown in table 1 . 
reality, many nutrients are required for successful growth, and animals have strategies to get the balance they need (Raubenheimer et al. 2009). The relative concentrations of different nutrients vary among plant parts, and further developments of our model should account for this.

\section{Conditions Determining Herbivore Strategies}

Prior state-dependent life-history models predicted that aposematic herbivores should grow more slowly and mature at a larger size than cryptic herbivores, as observed in a survey of Lepidoptera (Higginson and Ruxton 2010). Our model predicts this outcome when herbivores can also change location. Indeed, color and location should often change at the same time. The increasing apparency of larger herbivores, combined with the effect of size on warningsignal effectiveness, implies that OCC is expected when the overall strategy is cryptic-when-small and aposematism-whenlarge (Higginson and Ruxton 2010; our fig. 1). Herbivores that acquire costly toxins from their food are unlikely to be found exposed on flowers when small because they cannot be sufficiently defended. Being warningly colored and well defended makes it unnecessary to avoid detection by predators. Thus, OCC should be associated with florivory. Thus, the benefits of florivory for later instars may explain a substantial fraction of the prevalence of OCC among lepidopteran larvae: $20 \%$ of genera change from cryptic to aposematic, more than those that are aposematic from the first instar (16\%; Higginson and Ruxton 2010). Because flowers support faster growth and are sometimes not available to some species at some times, selection for florivory may explain the greater range of sizes observed among colorchanging species (Higginson and Ruxton 2010; A. D. Higginson, unpublished data). A survey of both color strategies and florivory among lepidopteran larvae would enable testing of our predictions.

The results suggest that the conditions necessary for herbivores to be cryptic on flowers are limited. This seems inconsistent with the occurrence of cryptic florivorous larvae across the Lepidoptera (McCall and Barr 2012; McCall et al. 2013; Tsuji and Sota 2013; Vargas 2014). However, limited occurrence in parameter space is not necessarily the same as uncommon environmental conditions. The fact that cryptic florivory is rare in parameter space but occurs in nature enables us to make strong predictions about the reasons that some florivores remain cryptic. If cryptic herbivores are equally difficult to detect on flowers and leaves, then cryptic florivory could evolve, yet there remains strong selection for a switch to aposematism in most of the parameter space. The selective pressure to evolve aposematism is weak only when toxins are very strong, leaves are much less nutritious and less well defended than flowers, and crypsis on leaves is highly effective. Thus, we predict these will be the conditions in which we observe cryptic florivory. Under such conditions OCC might evolve, but from a green leaf-cryptic form to a flower-cryptic form. Of course, given between-species diversity in flower appearance traits, this could occur only if the herbivore is highly specialized to a particular host or is polymorphic to match the flower colors of multiple species.

The model also predicts that cryptic florivores may sequester toxins for their defense, which may have been overlooked because of the expectation that toxic prey should be aposematic. This may be why many otherwise cryptic herbivores retain memorable patterns (e.g., of green, yellow, and white; Porter 1997) that may signal noxiousness. If defended cryptic florivores are common, they may even impose sufficient selection on plants for the evolution of between-species, within-species, or even within-individual variation in flower color.

We have assumed that the flowering period of the host plant is sufficiently long that flowers are always available to the focal herbivore during their ontogeny. This is realistic for temperate lepidopteran species, for example, that can cycle through several generations in a single summer and so be likely to complete at least one generation within the flowering period. This generation should be selected to follow the strategies we have identified. Our approach is conservative in terms of exploration of the optimal lifehistory constraints on exploitation of flowers, as a limited flowering period may constrain possible strategies. However, our predictions should also be relevant to specialist herbivores on plants with short flowering periods. If egg laying can be timed so that flowers are present when herbivores can benefit from exploiting these flowers, then the predicted strategies could still be observed. Indeed, the fitness advantages of florivory may provide selection for herbivores to temporally synchronize with particular hosts, leading to further specialization. We leave for future work the study of the selective pressures for synchrony of the herbivore life history with the flowering period.

\section{Coevolution of Herbivores and Their Host Plants}

Florivory is directly damaging to plant reproduction and so may heighten plant-herbivore coevolutionary antagonism. Plant defense strategies often involve responding to leaf damage by the production of toxins (Euler and Baldwin 1996; Adler et al. 2006; McCall and Karban 2006), which could increase the toxins available to specialized herbivores and so lead to more florivory, provided that the physiological link between leaf and flower toxin concentrations (Adler et al. 2012) is broken. The selective impact of herbivores on plant strategies may thus be even more complex than is currently appreciated (van Meijden 1996). The complexity is especially clear for aposematic herbivores (fig. $3 c$ ), for 
which fitness is highest at different leaf toxicities, depending on whether flowers are present. This raises the intriguing possibility that - when plants have evolved with a specialist herbivore - their leaf defense strategies may depend on whether they are in flower and so may change over the annual cycle.

Interestingly, our model predicts that aposematic florivory is unlikely to occur for very strong toxins (fig. $5 e$ ), because it is too costly for the herbivore to be well defended, but, of course, in natural systems such toxins may be too costly for the plant to maintain (McCall and Fordyce 2010). Physiological or ecological constraints on the production of secondary defenses will mean that plants' options are subject to a trade-off between the fitness costs of florivory and folivory. Plant defense strategies may therefore not minimize leaf consumption but may be selected to alter the amount of toxins in leaves or even the effectiveness of toxins during flowering, to reduce florivory. The preference of herbivores for flowers could conceivably be advantageous to plants. Flowers that are more conspicuous are expected to be better defended (McCall and Irwin 2006), but this may be less necessary because florivores will be more apparent to predators than folivores. Thus, the conspicuousness of flowers may reduce the consumption of all tissues.

We predict that in some situations defensive strategies slow herbivore growth or otherwise reduce herbivore fitness, whereas in others herbivores should mature sooner. These changes will have complex effects on herbivore population growth rates while also affecting the intensity of florivory. Because the potential population growth rate of the herbivore is affected by the plant's defenses, there may be a trade-off between damage to the individual plant and herbivory on the individual's offspring. Plant defense strategies may thus be influenced by the duration of the herbivore's lifetime relative to that of the plant. Evolved plant strategies will depend on the balance between individual and descendent reproductive success, the dispersal rates of plants and herbivores, and an evolutionary game among plants. It is also crucially important to consider predators of herbivores when studying plant defenses. Full understanding of the effects of herbivores on plant defense will require an evolutionary approach to tritrophic interactions that explicitly allows the change in plant strategy to be driven by the strategy of the focal herbivore. Here, we have demonstrated that florivory is likely to be widespread among aposematic small herbivores, especially later during their growth period, and so florivory is likely to be an important component of these widespread ecological interactions.

\section{Acknowledgments}

The authors are grateful to T. Day, L. Harder, and two anonymous reviewers for their insightful comments on an earlier draft of this article. A.D.H. was supported by the European Research Council (Advanced Grant 250209 to A. Houston) and fellowships from the Wissenschaftskolleg zu Berlin and the Natural Environment Research Council (NE/L011921/1).

\section{Literature Cited}

Adler, L. S., M. G. Seifert, M. Wink, and G. E. Morse. 2012. Reliance on pollinators predicts defensive chemistry across tobacco species. Ecology Letters 15:1140-1148.

Adler, L. S., M. Wink, M. Distl, and A. J. Lentz. 2006. Leaf herbivory and nutrients increase nectar alkaloids. Ecologv Letters 9:960-967.

Agerbirk, N., F. S. Chew, C. E. Olsen, and K. Jorgensen. 2010. Leaf and floral parts feeding by orange tip butterfly larvae depends on larval position but not on glucosinolate profile or nitrogen level. Journal of Chemical Ecology 36:1335-1345.

Bandeili, B., and C. Müller. 2010. Folivory versus florivoryadaptiveness of flower feeding. Naturwissenschaften 97:79-88.

Benrey, B., and R. F. Denno. 1997. The slow-growth-high-mortality hypothesis: a test using the cabbage butterfly. Ecology 78:987-999.

Berger, D., R. Walters, and K. Gotthard. 2006. What keeps insects small? size dependent predation on two species of butterfly larvae. Evolutionarv Ecology 20:575-589.

Bowers, M. D. 1992. The evolution of unpalatability and the cost of chemical defense in insects. Pages 216-244 in B. D. Roitberg and M. B. Isman, eds. Insect chemical ecology: an evolutionary approach. Chapman \& Hall, London.

- 1993. Aposematic caterpillars: life-styles of the warningly colored and unpalatable. Pages 331-371 in N. E. Stamp and T. M. Casey, eds. Caterpillars: ecological and evolutionary constraints on foraging. Chapman \& Hall, London.

Cott, H. B. 1940. Adaptive coloration in animals. Methuen, London. Daly, D., A. D. Higginson, C. Dong, G. D. Ruxton, and M. P. Speed. 2012. Density-dependent investment in costly antipredator defences: an explanation for the weak survival benefit of group living. Ecology Letters 15:576-583.

Damman, H. 1987. Leaf quality and enemy avoidance by the larvae of a pyralid moth. Ecology 68:88-97.

Diniz, I. R., and H. C. Morais. 2002. Local pattern of host plant utilization by lepidopteran larvae in the cerrado vegetation. Entomotropica 17:115-119.

Eccard, J. A., and T. Liesenjohann. 2014. The importance of predation risk and missed opportunity costs for context-dependent foraging patterns. PLoS ONE 9:e94107. doi:10.1371/journal.pone.0094107.

Euler, M., and I. T. Baldwin. 1996. The chemistry of defense and apparency in the corollas of Nicotiana attenuata. Oecologia (Berlin) 107:102-112.

Forsman, A., and S. Merilaita. 1999. Fearful symmetry: pattern size and asymmetry affects aposematic signal efficacy. Evolutionary Ecology 13:131-140.

Grant, J. B. 2006. Diversification of gut morphology in caterpillars is associated with defensive behavior. Lournal of Experimental Biology 209:3018-3024.

. 2007. Ontogenetic colour change and the evolution of aposematism: a case study in panic moth caterpillars. Iournal of Animal Ecology 76:439-447.

Hall, J. R., I. C. Cuthill, R. Baddeley, A. J. Shohet, and N. E. ScottSamuel. 2013. Camouflage, detection and identification of moving 
targets. Proceedings of the Roval Society B: Biological Sciences 280:20130064. doi:10.1098/rspb.2013.0064.

Heinrich, B. 1979. Foraging strategies of caterpillars. Oecologia (Berlin) 42:325-337.

Heinrich, B., and S. L. Collins. 1983. Caterpillar leaf damage, and the game of hide-and-seek with birds. Ecology 64:592-602.

Held, D. W., and D. A. Potter. 2004. Floral affinity and benefits of dietary mixing with flowers for a polyphagous scarab, Popillia japonica Newman. Oecologia (Berlin) 140:312-320.

Herms, D. A., and W. J. Mattson. 1992. The dilemma of plants: to grow or defend. Quarterly Review of Biology 67:283-335.

Higginson, A. D., J. Delf, G. D. Ruxton, and M. P. Speed. 2011. Growth and reproductive costs of larval defence in the aposematic lepidopteran Pieris brassicae. Journal of Animal Ecology 80:384392.

Higginson, A. D., and G. D. Ruxton. 2009. Dynamic state-dependent modelling predicts optimal usage patterns of responsive defences. Oecologia (Berlin) 160:399-410.

2010. Optimal defensive coloration strategies during the growth period of prey. Evolution 64:53-67.

Higginson, A. D., M. P. Speed, and G. D. Ruxton. 2012. Effects of anti-predator defence through toxin sequestration on use of alternative food microhabitats by small herbivores. ical Biology 300:368-375.

Houston, A. I., and J. M. McNamara. 1999. Models of adaptive behaviour. Cambridge University Press, Cambridge.

Ioannou, C. C., and J. Krause. 2009. Interactions between background matching and motion during visual detection can explain why cryptic animals keep still. Biology Letters 5:191-193.

Kursar, T. A., and P. D. Coley. 1992. Delayed greening in tropical leaves: an anti-herbivore defence? Biotropica 24:256-262.

Mänd, T., T. Tammaru, and J. Mappes. 2007. Size dependent predation risk in cryptic and conspicuous insects. Evolutionary Ecology 21:485-498.

Mäntylä, E., T. Klemola, P. Sirkiä, and T. Laaksonen. 2008. Low light reflectance may explain the attraction of birds to defoliated trees. Behavioral Ecology 19:325-330.

Mappes, J., N. M. Marples, and J. A. Endler. 2005. The complex business of survival by aposematism. Trends in Ecology and Evolution 20:598-603.

McCall, A. C., and C. M. Barr. 2012. Why do florivores prefer hermaphrodites over females in Nemophila menziesii (Boraginaceae)? Oecologia (Berlin) 170:147-157.

McCall, A. C., and J. A. Fordyce. 2010. Can optimal defence theory be used to predict the distribution of plant chemical defences? Journal of Ecology 98:985-992.

McCall, A. C., and R. E. Irwin. 2006. Florivory: the intersection of pollination and herbivory. Ecology Letters 9:1351-1365.

McCall, A. C., and R. Karban. 2006. Induced defense in Nicotiana attenuata (Solanaceae) fruit and flowers. Oecologia (Berlin) 146: 566-571.

McCall, A. C., S. J. Murphy, C. Venner, and M. Brown. 2013. Florivores prefer white versus pink petal color morphs in wild radish, Raphanus sativus. Oecologia (Berlin) 172:189-195.

McNamara, J. M. 1990. The policy which maximizes long-term survival of an animal faced with the risks of starvation and predation. Advances in Applied Probability 22:295-308.

Morais, H. C., J. Du Vall Hay, and I. R. Diniz. 2009. Brazilian cerrado folivore and florivore caterpillars: how different are they? Biotropica 41:401-405.
Nonacs, P. 2001. State dependent behavior and the marginal value theorem. Behavioral Ecology 12:71-83.

Opitz, S. E. W., and C. Müller. 2009. Plant chemistry and insect sequestration. Chemoecology 19:117-154.

Porter, J. 1997. The colour identification guide to the caterpillars of the British Isles. Viking, London.

Poulton, E. B. 1890. The colours of animals. Trübner, London.

Raubenheimer, D., S. J. Simpson, and D. Mayntz. 2009. Nutrition, ecology and nutritional ecology: toward an integrated framework. Functional Ecology 23:4-16.

Reader, T., and D. F. Hochuli. 2003. Understanding gregariousness in a larval lepidopteran: the roles of host plant predation and microclimate. Ecological Entomology 28:729-737.

Remmel, T., and T. Tammaru. 2011. Evidence for the higher importance of signal size over body size in aposematic signaling in insects. Journal of Insect Science 11:4. doi:10.1673/031.011.0104.

Reynolds, S. E., S. F. Nottingham, and A. E. Stephens. 1985. Food and water economy and its relation to growth in fifth-instar larvae of the tobacco hornworm, Manduca sexta. Journal of Insect Physiology 31:119-127.

Ruxton, G. D., T. N. Sherratt, and M. P. Speed. 2004. Avoiding attack. Oxford University Press, Oxford.

Singer, M. S., Y. Carrière, C. Theuring, and T. Hartmann. 2004a. Disentangling food quality from resistance against parasitoids: diet choices by a generalist caterpillar. American Naturalist 164: 423-429.

Singer, M. S., D. Rodrigues, J. O. Stireman, and Y. Carrière. $2004 b$ Roles of food quality and enemy-free space in host use by a generalist insect herbivore. Ecology 85:2747-2753.

Skelhorn, J., H. M. Rowland, M. P. Speed, and G. D. Ruxton. 2010. Masquerade: camouflage without crypsis. Science 327:51.

Smallegange, R. C., J. J. A. van Loon, S. E. Blatt, J. A. Harvey, N. Agerbirk, and M. Dicke. 2007. Flower vs. leaf feeding by Pieris brassicae: glucosinolate-rich flower tissues are preferred and sustain higher growth rate. Journal of Chemical Ecology 33:1831-1844. Speed, M. P., M. A. Brockhurst, and G. D. Ruxton. 2010. The dual benefits of aposematism: predator avoidance and enhanced resource collection. Evolution 64:1622-1633.

Stamp, N. E., and M. D. Bowers. 1988. Direct and indirect effects of predatory wasps (Polistes sp.: Vespidae) on gregarious caterpillars (Hemileuca lucina: Saturnidae). Oecologia (Berlin) 75:619-624.

. 1991. Indirect effect on survivorship of caterpillars due to presence of invertebrate predators. Oecologia (Berlin) 88:325-330.

Stevens, M., and G. D. Ruxton. 2013. Linking the evolution and form of warning coloration in nature. Proceedings of the Roval Society B: Biological Sciences 279:417-426.

Takeuchi, H., M. P. Zalucki, and M. J. Furlong. 2009. Crocidolomia pavonana larval foraging: behavior and feeding site preferences on cabbage Brassica oleracea. Entomologia Experimentalis et Applicata 133:154-164.

Tammaru, T., and T. Esperk. 2007. Growth allometry of immature insects: larvae do not grow exponentially. Functional Ecology 21: 1099-1105.

Tsuji, K., and T. Sota. 2013. Florivores on the dioecious shrub Eurya japonica and the preferences and performances of two polyphagous geometrid moths on male and female plants. Entomological Science 16:291-297.

van Dam, N. M., T. J. de Jong, Y. Iwasa, and T. Kubo. 1996. Optimal distribution of defences: are plants smart investors? Functional Ecology 10:128-136. 
van Meijden, E. 1996. Plant defence, an evolutionary dilemma: contrasting effects of (specialist and generalist) herbivores and natural enemies. Entomologia Experimentalis et Applicata 80:307-310.

Vargas, H. A. 2014. Lycaenid caterpillars (Lepidoptera, Lycaenidae) eating flowers of Dalea pennellii var. chilensis (Fabaceae) in the northern Chilean Andes. Revista Brasileira de Entomologia 58:309_ 312 .

Werner, E. E., and J. F. Gilliam. 1984. The ontogenetic niche shift and species interactions in size-structured populations. Annual Review of Ecology and Systematics 15:393-425.
Williams, I. S. 1999. Slow-growth, high-mortality - a general hypothesis, or is it? Ecological Entomology 24:490-495.

Wilson, D., R. Heinsohn, and J. A. Endler. 2007. The adaptive significance of ontogenetic colour change in a tropical python. Biology Letters 3:40-43.

Winterhalder, B. 1983. Opportunity-cost foraging models for stationary and mobile predators. American Naturalist 122:73-84.

Associate Editor: Lawrence D. Harder Editor: Susan Kalisz

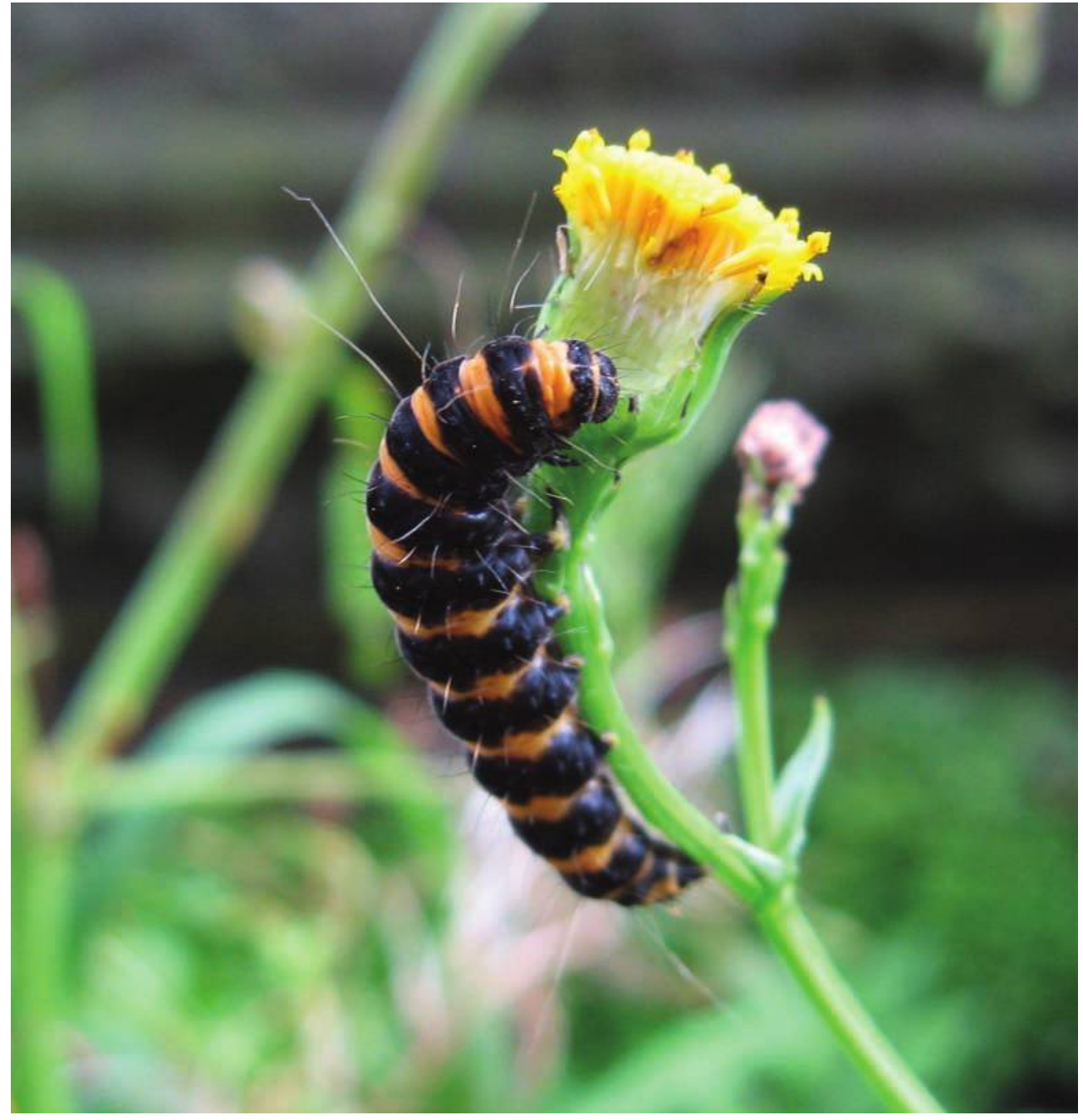

Cinnabar moth (Tyria jacobaeae) larva feeding on an inflorescence of ragwort (Senecio spp.) in an urban garden in northeastern England. Tyria jacobaeae sequesters pyrrolizidine alkaloids from its host plant that make it unpalatable to predators, so it is able to feed conspicuously on flowers. Photograph by A. D. Higginson. 